\title{
Effect of thermal annealing treatment and defect analysis on
}

\section{$\mathrm{AgGaGeS}_{4}$ single crystal}

Wei Huang, Zhiyu He*, Beijun Zhao, Shifu Zhu, Baojun Chen, Ying Wu

College of Materials Science and Engineering, Sichuan University, Chengdu

\author{
610064, China
}

Corresponding author. Fax: +862885412745 .

E-mail address: hzyscu@163.com

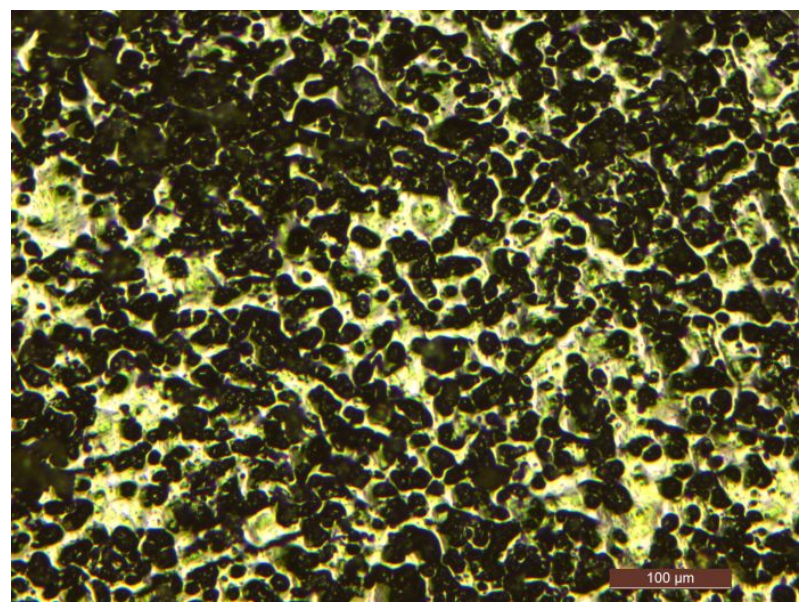

Figure S1. Metallographic microscope photographs of $\mathrm{AgGaGeS}_{4}$ wafer surface after annealing with polycrystalline powder at $600{ }^{\circ} \mathrm{C}$

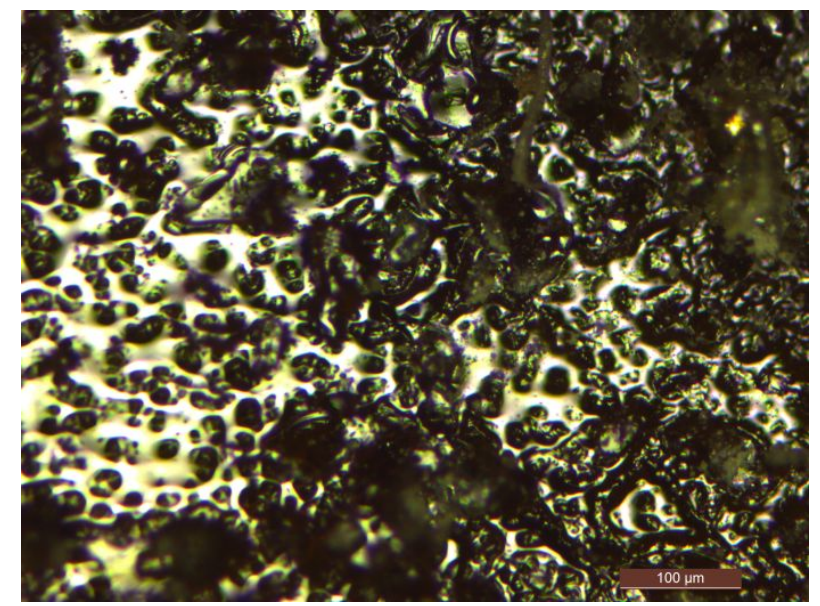

Figure S2. Metallographic microscope photographs of $\mathrm{AgGaGeS}_{4}$ wafer surface after annealing with polycrystalline powder at $650{ }^{\circ} \mathrm{C}$ 


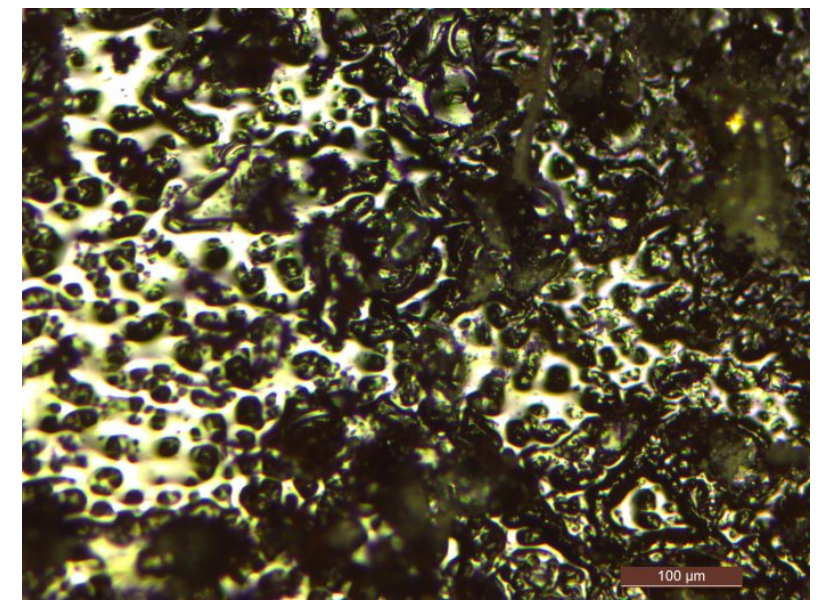

Figure S3. Metallographic microscope photographs of $\mathrm{AgGaGeS}_{4}$ wafer surface after annealing with polycrystalline powder at $700{ }^{\circ} \mathrm{C}$

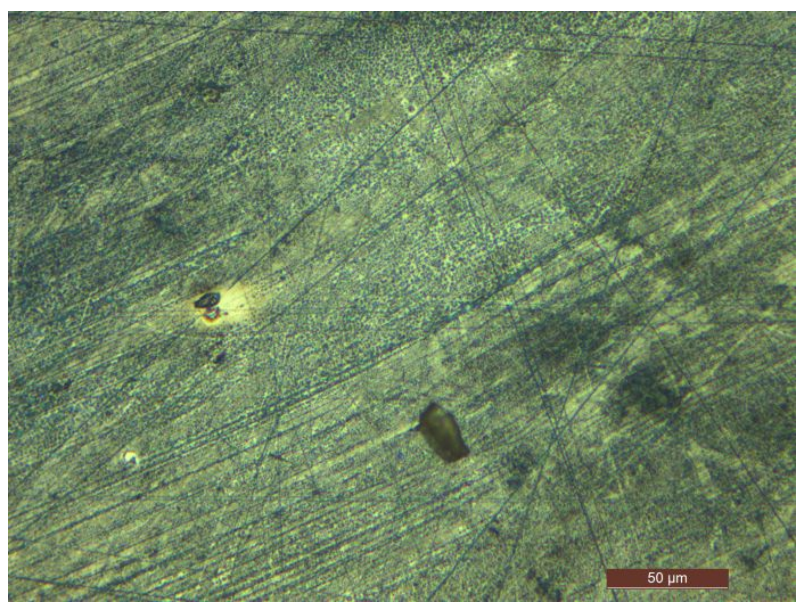

Figure S4. Metallographic microscope photographs of $\mathrm{AgGaGeS}_{4}$ wafer surface after annealing in vacuum at $500{ }^{\circ} \mathrm{C}$

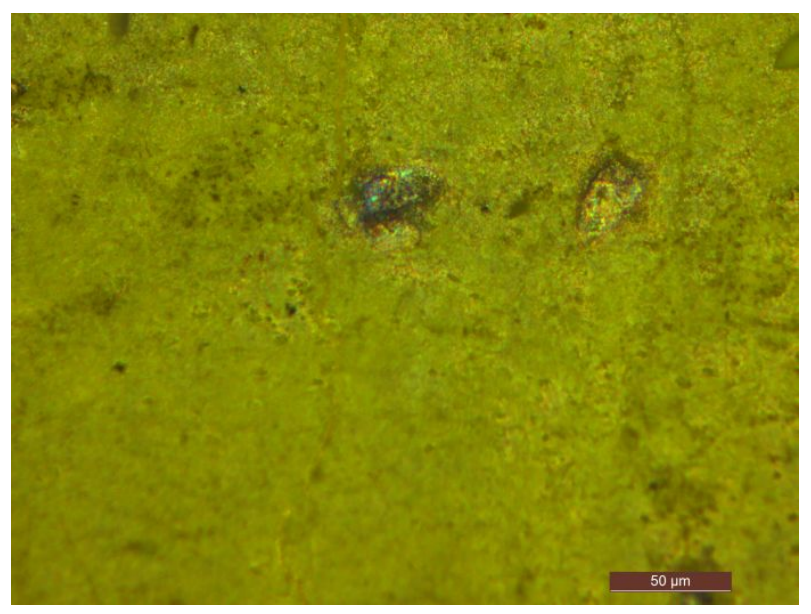

Figure S5. Metallographic microscope photographs of $\mathrm{AgGaGeS}_{4}$ wafer surface after annealing in vacuum at $600{ }^{\circ} \mathrm{C}$ (additional experiment) 

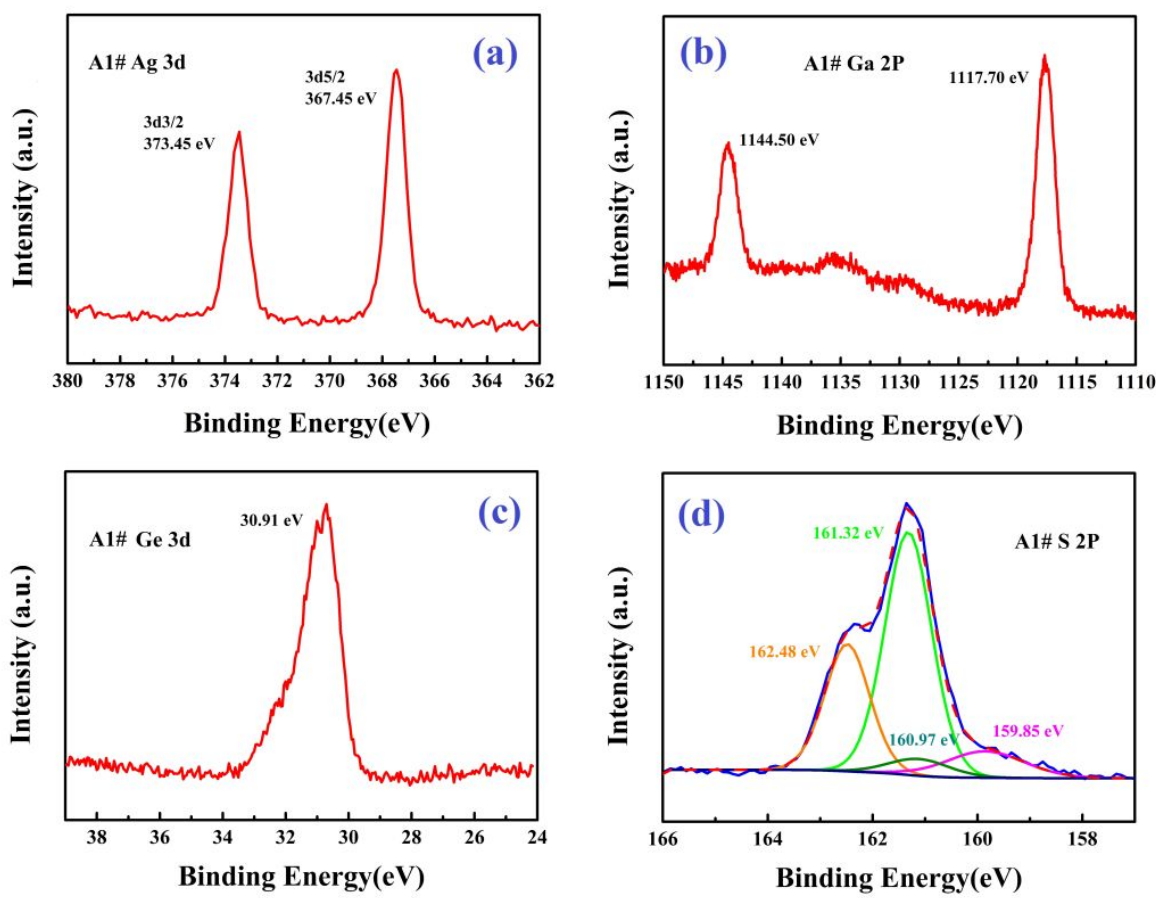

Figure S6. Detailed XPS recorded for polished surfaces of the A1\# $\mathrm{AgGaGeS}_{4}$ single crystals (a) Ag 3d; (b) Ga 2p; (c) Ge 3d; (d) S 2p
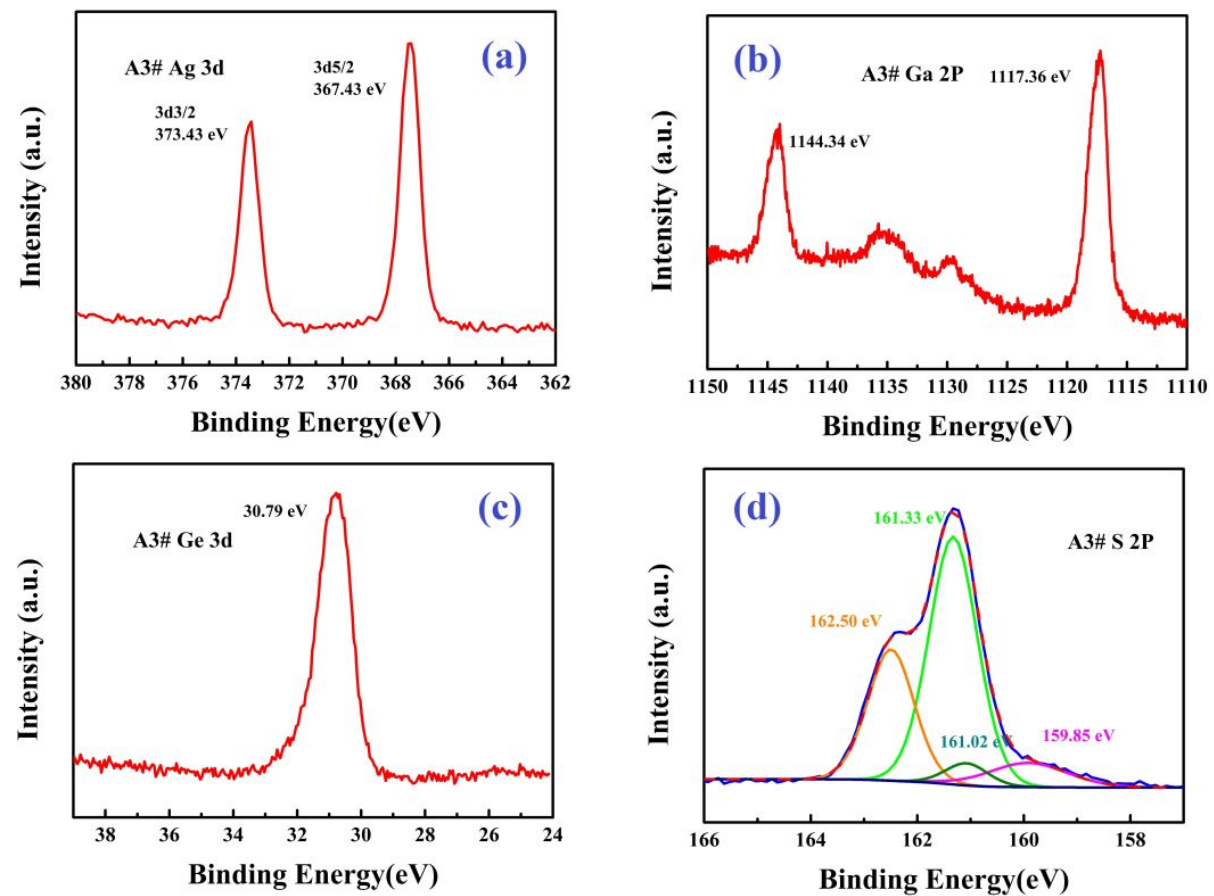

Figure S7. Detailed XPS recorded for polished surfaces of the A3\# $\mathrm{AgGaGeS}_{4}$ single crystals (a) Ag 3d; (b) Ga 2p; (c) Ge 3d; (d) S 2p 

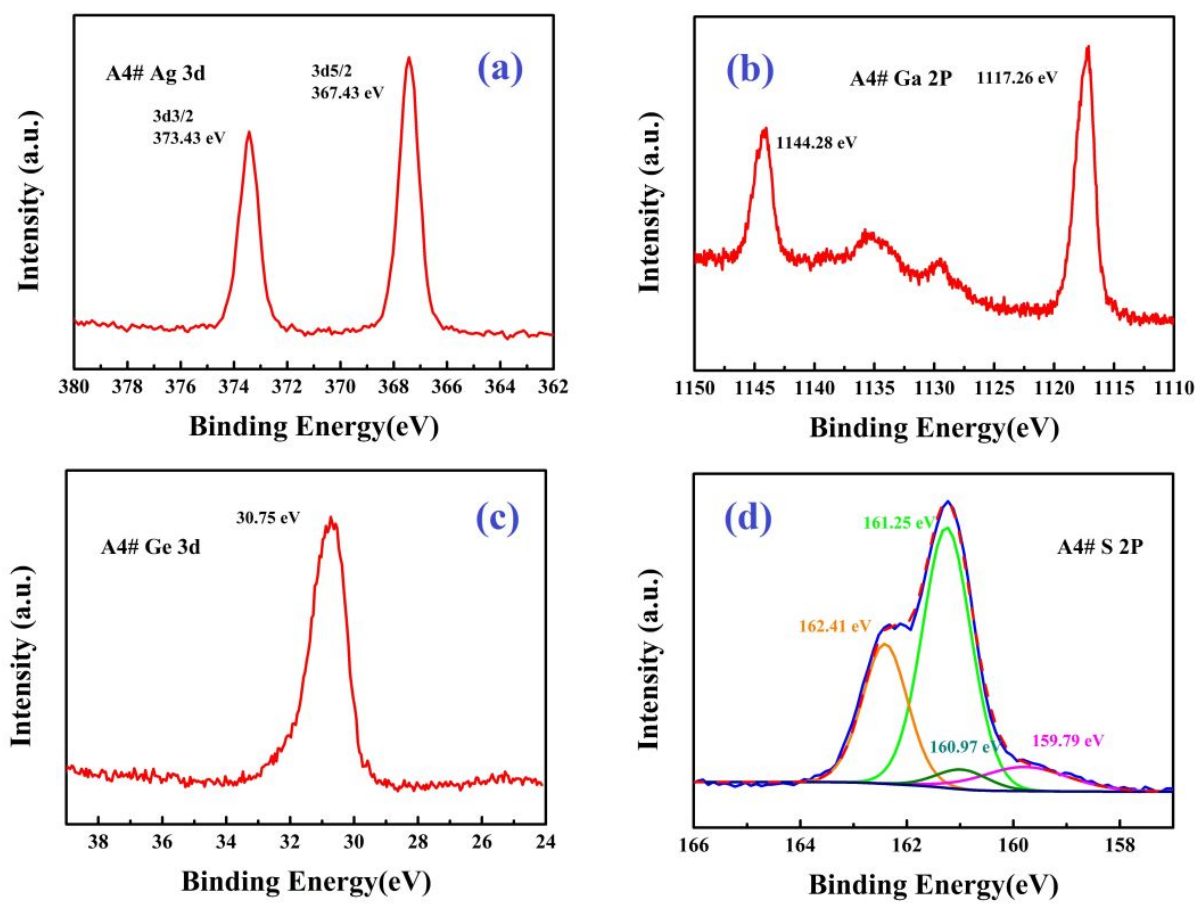

Figure S8. Detailed XPS recorded for polished surfaces of the A4\# $\mathrm{AgGaGeS}_{4}$ single crystals (a) Ag 3d; (b) Ga 2p; (c) Ge 3d; (d) S 2p
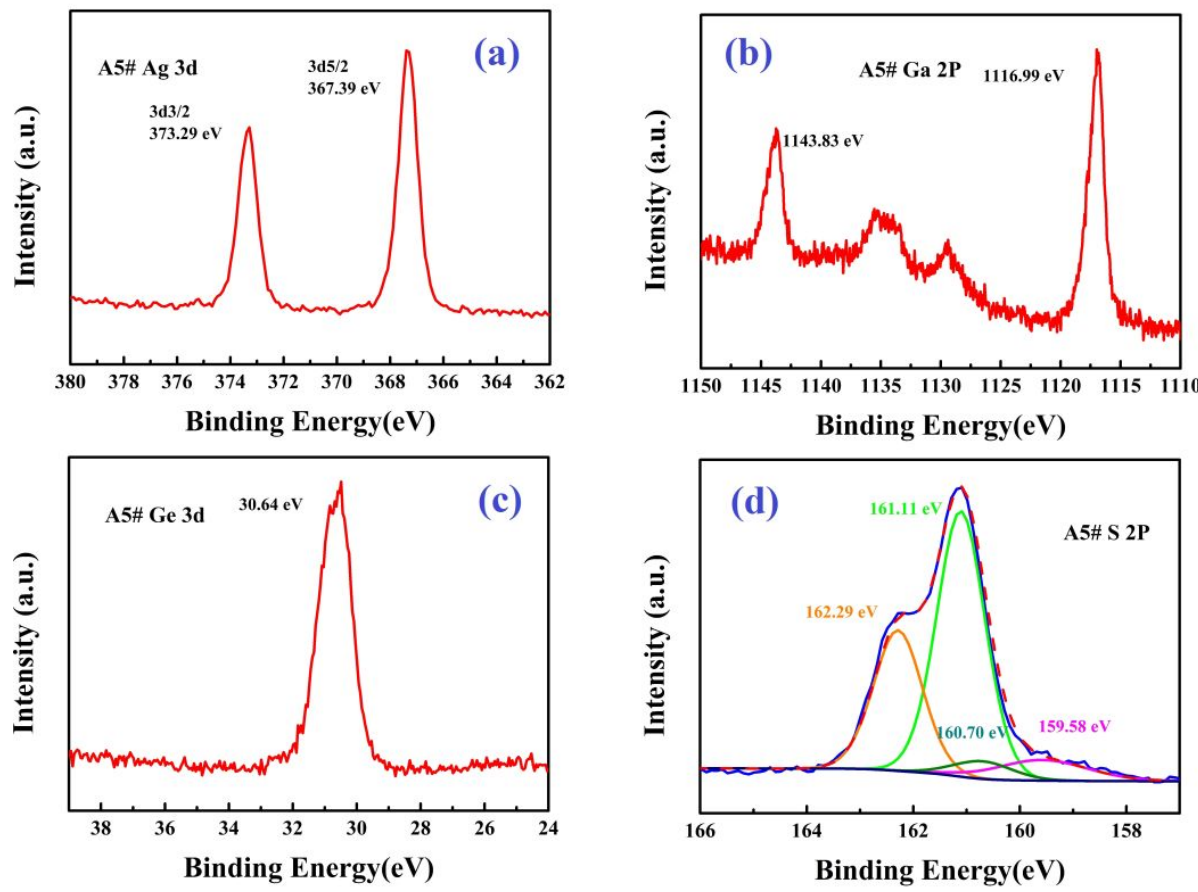

Figure S9. Detailed XPS recorded for polished surfaces of the A5\# $\mathrm{AgGaGeS}_{4}$ single crystals (a) Ag 3d; (b) Ga 2p; (c) Ge 3d; (d) S 2p 

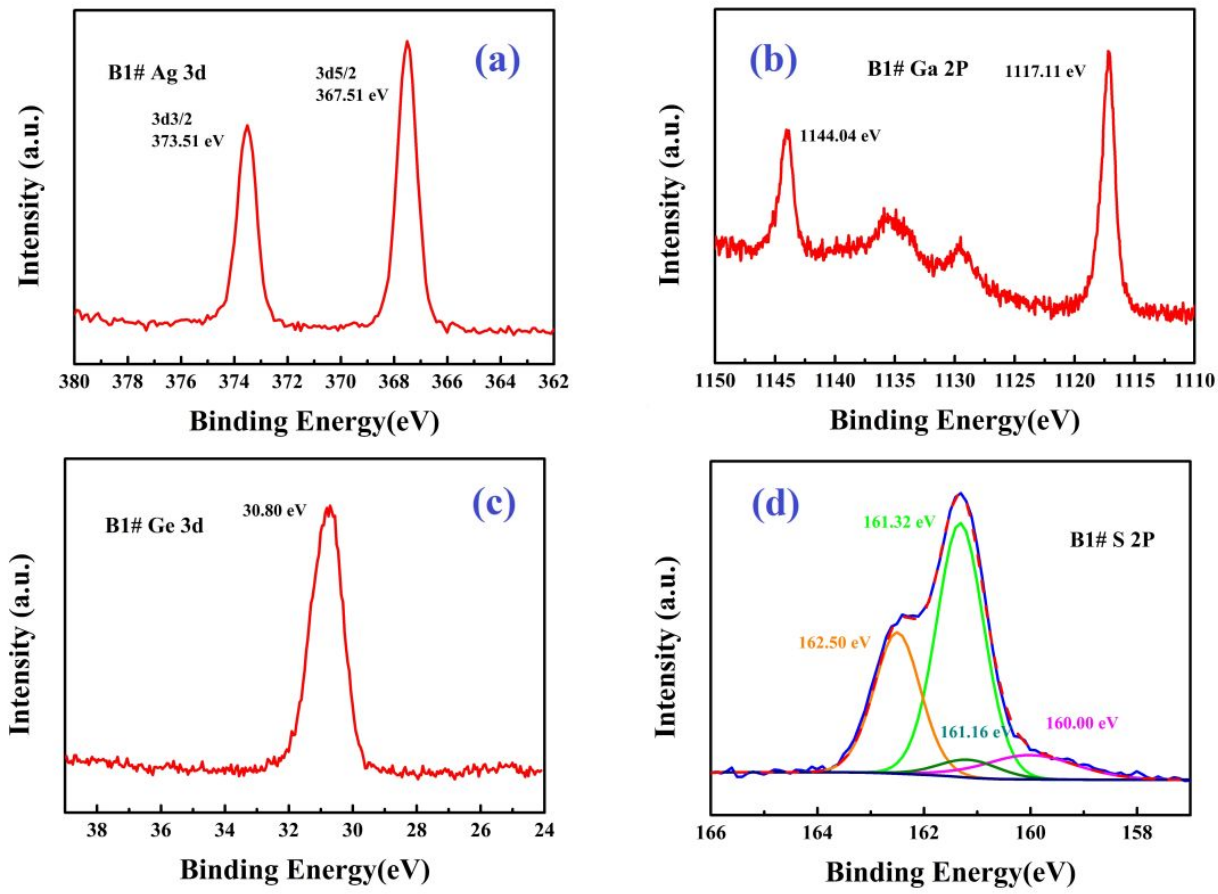

Figure S10. Detailed XPS recorded for polished surfaces of the B1\# $\mathrm{AgGaGeS}_{4}$ single crystals (a) Ag 3d; (b) Ga 2p; (c) Ge 3d; (d) S 2p
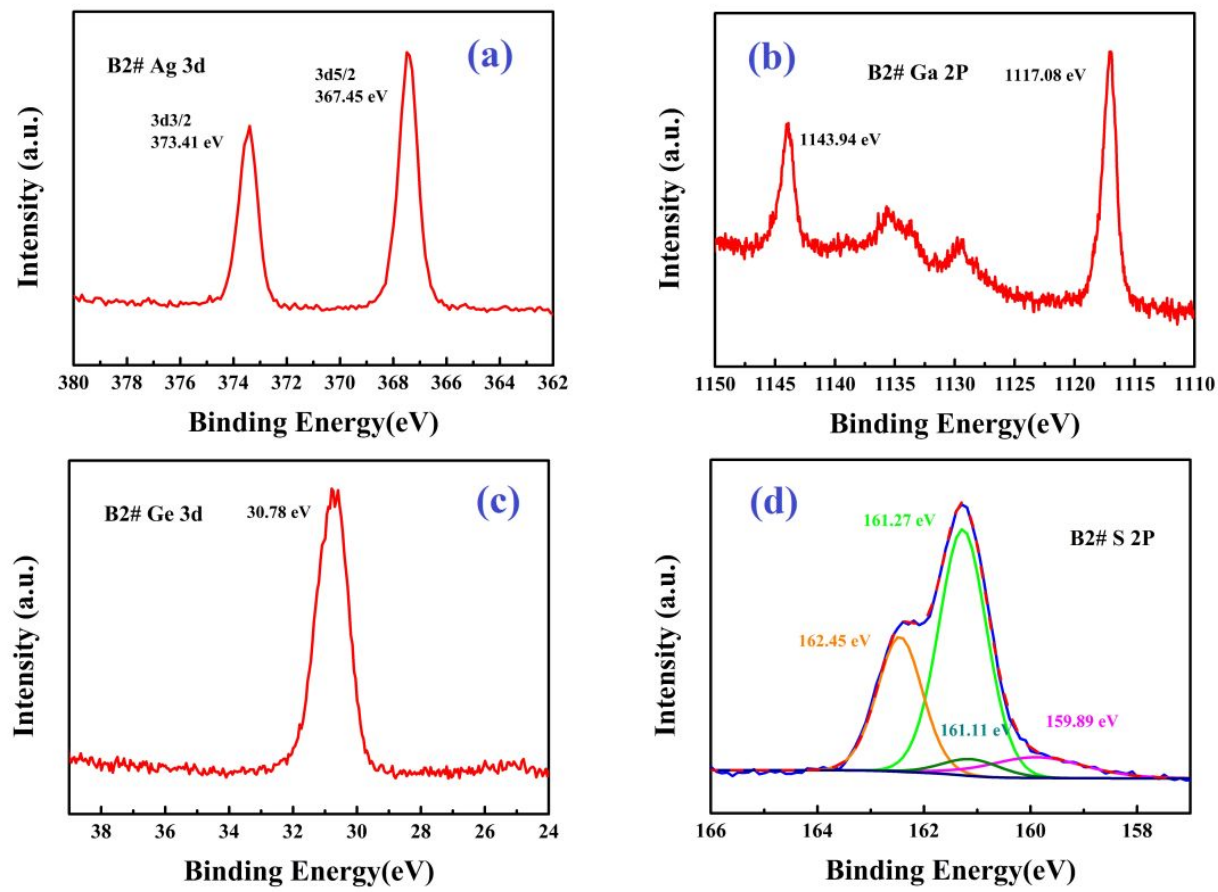

Figure S11. Detailed XPS recorded for polished surfaces of the B2\# $\mathrm{AgGaGeS}_{4}$ single crystals (a) Ag 3d; (b) Ga 2p; (c) Ge 3d; (d) S 2p 

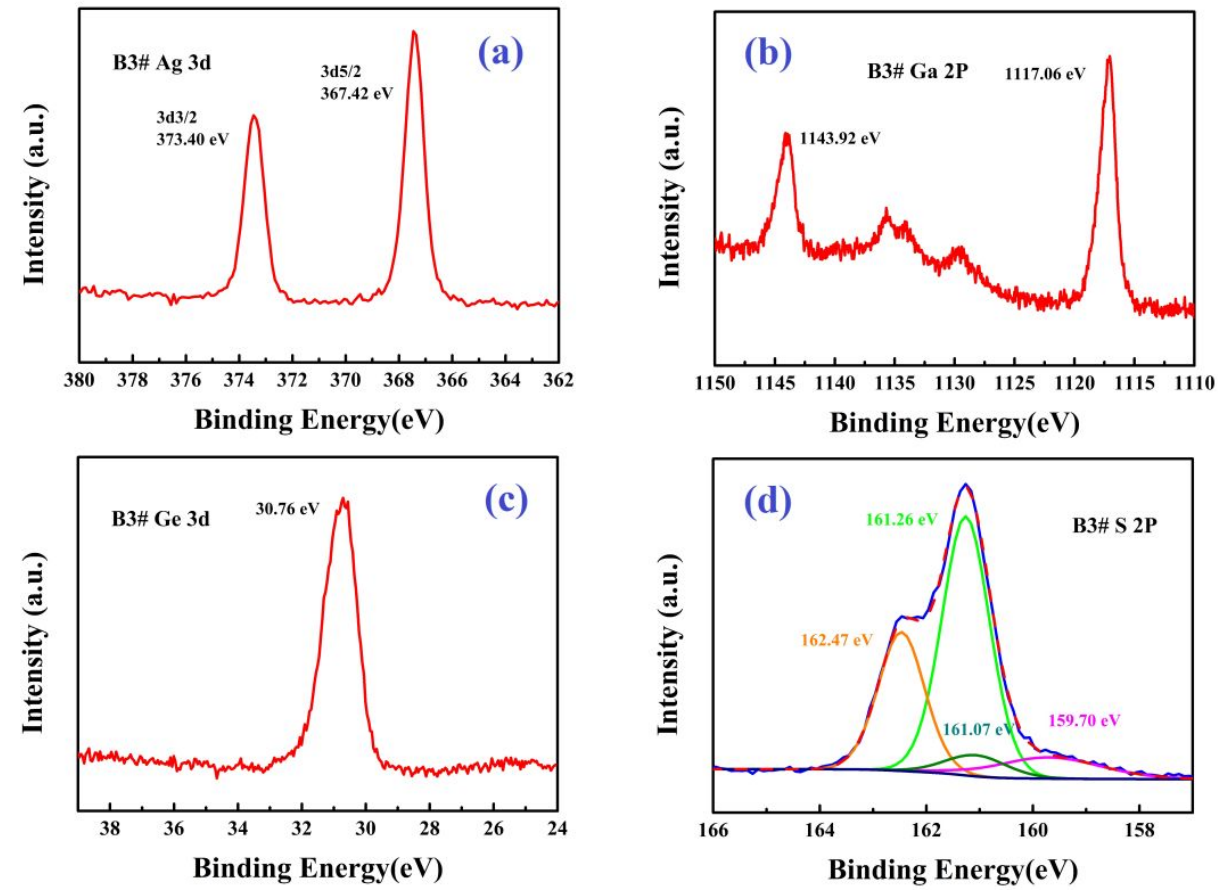

Figure S12. Detailed XPS recorded for polished surfaces of the B3\# $\mathrm{AgGaGeS}_{4}$ single crystals (a) Ag 3d; (b) Ga 2p; (c) Ge 3d; (d) S 2p
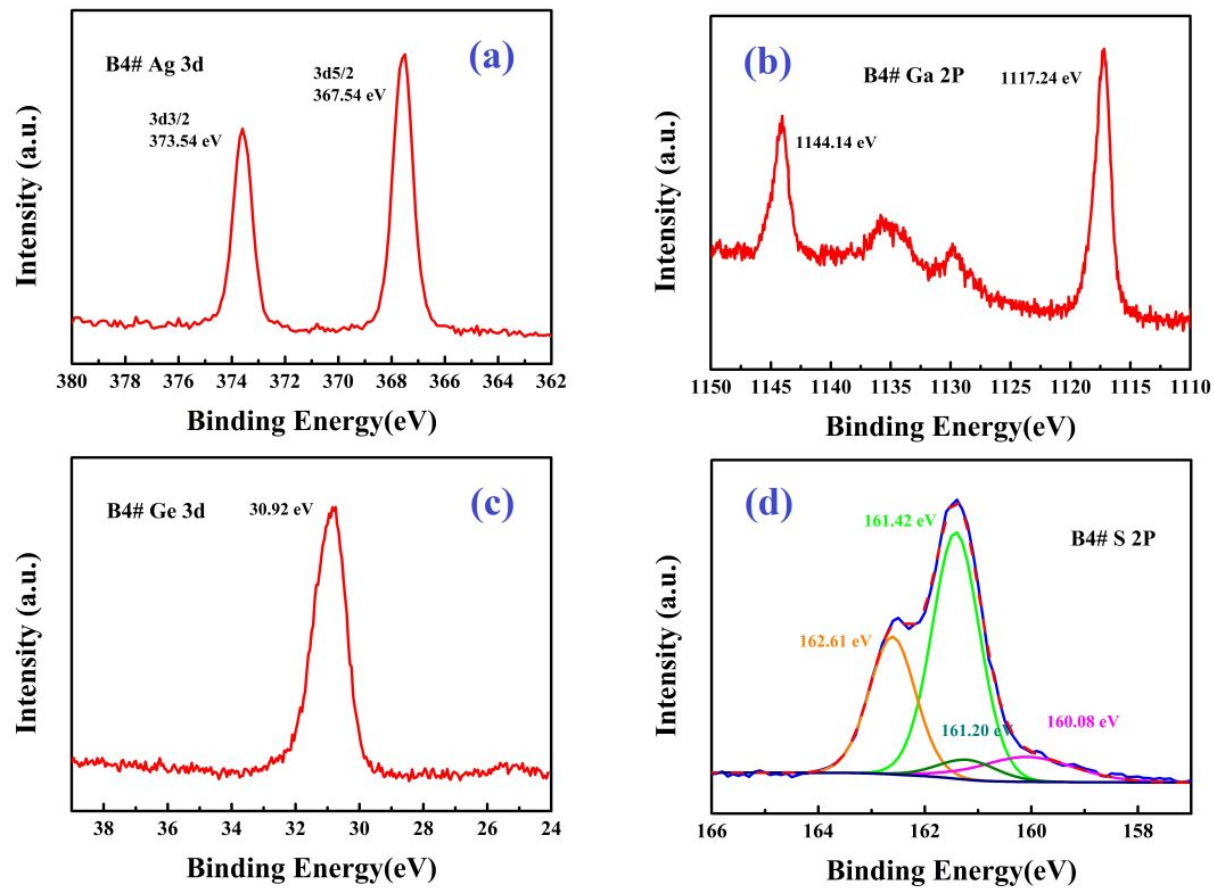

Figure S13. Detailed XPS recorded for polished surfaces of the B4\# $\mathrm{AgGaGeS}_{4}$ single crystals (a) Ag 3d; (b) Ga 2p; (c) Ge 3d; (d) S 2p 

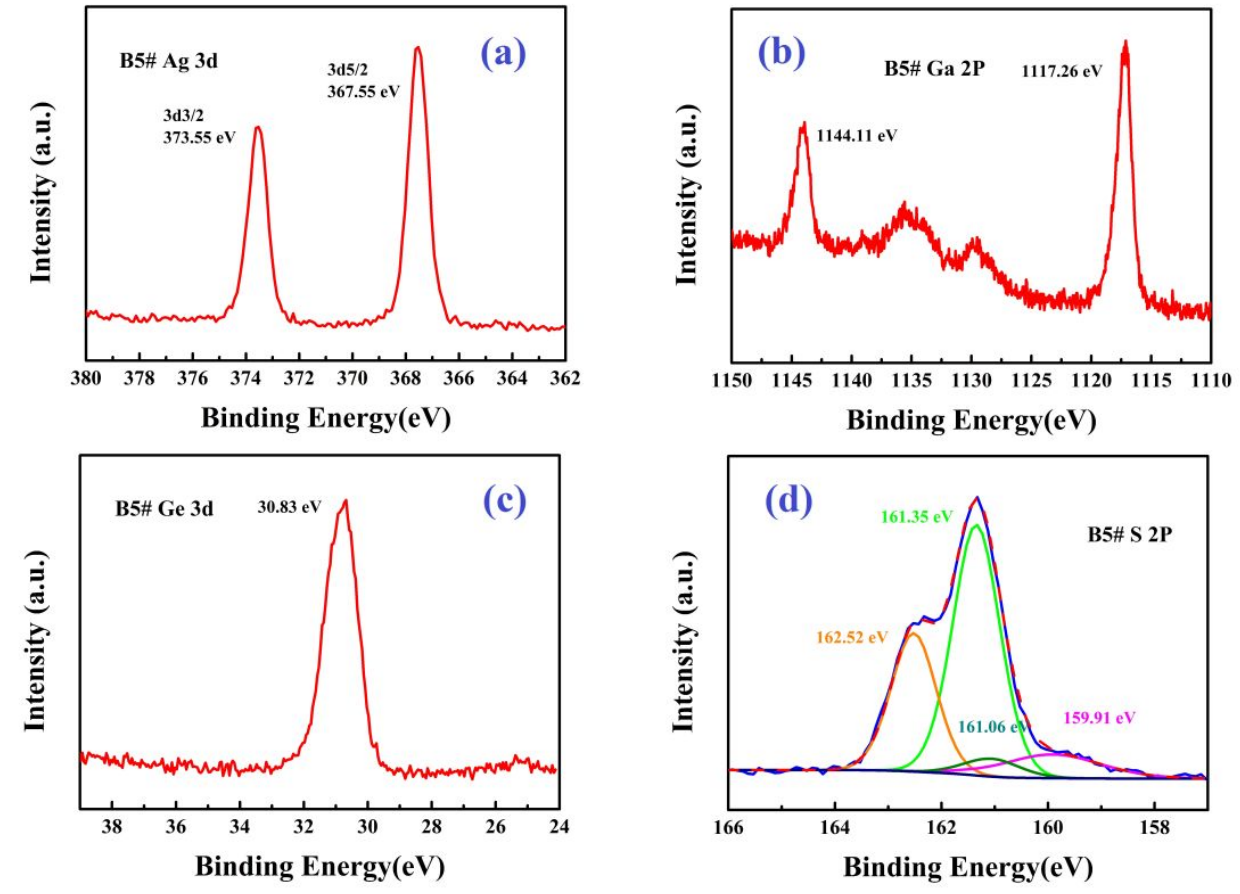

Figure S14. Detailed XPS recorded for polished surfaces of the B5\# $\mathrm{AgGaGeS}_{4}$ single crystals (a) Ag 3d; (b) Ga 2p; (c) Ge 3d; (d) S 2p 